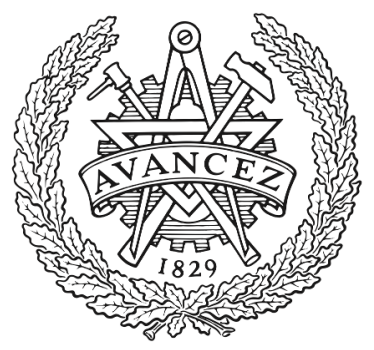

CHALMERS

UNIVERSITY OF TECHNOLOGY

\title{
Regenerator Site Predeployment in Nonlinear Dynamic Flexible-Grid Networks
}

Downloaded from: https://research.chalmers.se, 2023-04-26 07:08 UTC

Citation for the original published paper (version of record):

Yan, L., Xu, Y., Brandt-Pearce, M. et al (2017). Regenerator Site Predeployment in Nonlinear Dynamic Flexible-Grid Networks. European Conference on Optical Communication, ECOC. http://dx.doi.org/10.1109/ECOC.2017.8345997

N.B. When citing this work, cite the original published paper. 


\title{
Regenerator Site Predeployment in Nonlinear Dynamic Flexible-Grid Networks
}

\author{
Li Yan(1), Yuxin Xu(2), Maïté Brandt-Pearce(2), Nishan Dharmaweera(1), and Erik Agrell(1)
}

(1) Chalmers University of Technology, lyaa@chalmers.se ${ }^{(2)}$ University of Virginia

\begin{abstract}
A regenerator predeployment algorithm is proposed in dynamic translucent flexible-grid networks based on the GN model. The randomness of traffic bandwidth requests is exploited to allocate regenerators efficiently. Our method accommodates $30 \%$ more demands than benchmark methods.
\end{abstract}

\section{Introduction}

The advent of colorless directionless reconfigurable optical add/drop multiplexers (CDROADMs) and software-defined optical networking can support dynamic control, management, and optimization of flexible-grid networks ${ }^{1}$, where sparse regenerator sites can be predeployed to enable fast provisioning of services and reconfigurations $^{2}$. To allocate regenerator site (RS) conservatively and effectively, it is necessary to accurately estimate the physical layer impairments (PLIs). Transmission reach (TR) is used in many previous studies $^{1,2}$ to guarantee proper quality of transmissions (QoTs). Recently, a more sophisticated RS placement ${ }^{3}$ algorithm based on the Gaussian noise (GN) model ${ }^{4}$ was proposed to reduce the number of RSs. However, most of the existing algorithms are based on the predicted static traffic demands, whereas the actual bandwidth requests are usually dynamic and random, which can be very different from the predicted data. Therefore, the randomness of traffic demands should be considered to allocate RS efficiently.

In this paper, we propose a new RS placement algorithm for random traffic demands in flexiblegrid networks based on the GN model. Assuming a predefined routing scheme, the method obtains the likelihood of being an RS for each network node using Monte Carlo simulations, based on which the RSs are selected.

\section{Problem Statement}

The goal is to allocate a limited number of RSs such that the blocking probability (BP) due to the lack of regeneration is minimized in a dynamic flexible-grid network. The network topology is represented as $(V, E)$, where $V$ is the set of CDROADM nodes and $E$ is the set of bidirectional links with equal-length fiber spans. The set of RSs is defined as $R \subset V$. The modulation format is used for all the traffic demands. To focus mainly on the PLI-related traffic blocking, unlimited spectrum resources are assumed.

To minimize the communication delay or cost per demand in carrier-grade networks, the shortest-path routing in terms of a certain criterion, e.g., the distance or light path cost, is preferred by all the traffic demands ${ }^{2}$. Constrained by this shortest-path requirement, the light paths for all the possible node pairs are thus determined $^{2}$. Therefore, we assume a predefined routing scheme when assigning RSs.

\section{Proposed Algorithm}

The proposed RS allocation algorithm is composed of two stages: we first allocate RSs in Monte Carlo simulations with random bandwidth demands, and then calculate the likelihood of each network node being an RS, according to which permanent RSs are placed to minimize the possible traffic blocking. The algorithm is summarized by a flowchart in Fig. 1 .

In the first stage, the statistical network assessment process (SNAP) framework ${ }^{5}$ is used to simulate the PLIs generated by random traffic demands. In SNAP, the randomly generated traffic

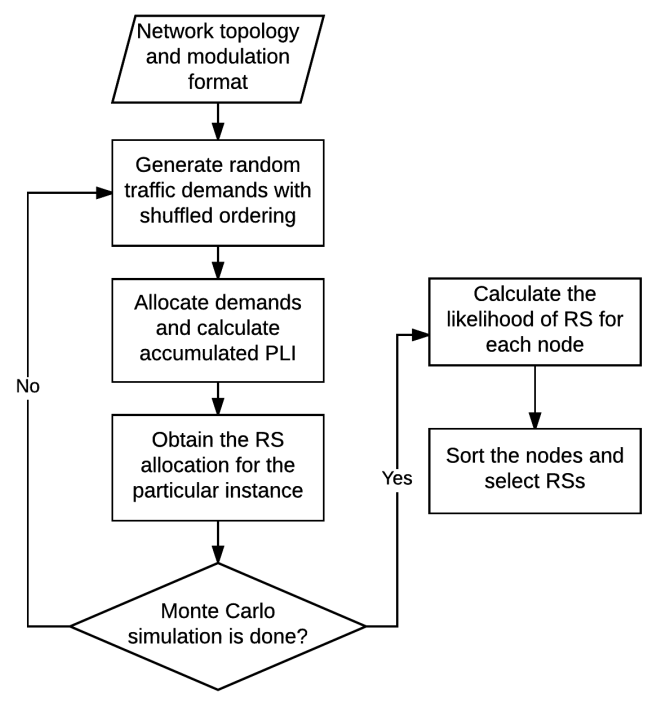

Fig. 1: The flowchart of the proposed algorithm. 
demands are shuffled and allocated in the network one by one. The first-fit policy is used for spectrum assignment. At the end of each simulation, the PLI of each traffic demand $d$ on link $l$, $G_{d, l}^{\mathrm{NL} \text {, }}$, is calculated by the $\mathrm{GN}$ model. The RS allocation regarding PLIs generated in this specific simulation instance is then optimized by (1).

$$
\begin{array}{ll}
\underset{N_{d, i}, C_{d, i}, I_{i}}{\operatorname{minimize}} \alpha C_{\text {total }}+\beta I_{\text {total }} & \\
C_{\text {total }}, I_{\text {total }} \\
\text { subject to } & \\
N_{d, i} \leq N_{d, \operatorname{src}(i ; d)}+L_{d, \operatorname{src}(i ; d), i} & \forall d \in D, i \in P_{d}, \\
N_{d, i} \leq M\left(1-C_{d, i}\right) & \forall d \in D, i \in P_{d}, \\
N_{d, \operatorname{src}(i ; d)}+L_{d, \operatorname{src}(i ; d), i}- & \forall d \in D, i \in P_{d}, \\
N_{d, i} \leq M C_{d, i} & \forall d \in D, i \in P_{d}, \\
N_{d, i} \geq 0 & \forall d \in D, i \in P_{d}, \\
N_{d, \operatorname{src}(i ; d)}+L_{d, \operatorname{src}(i ; d), i} \leq N_{\max } & \forall i \in V, \\
\sum_{d \in D} C_{d, i} \leq I_{i} C_{\max } & \\
C_{\text {total }}=\sum_{d \in D} \sum_{i \in P_{d}} C_{d, i}, & \\
I_{\text {total }}=\sum_{i \in V} I_{i} . &
\end{array}
$$

The parameters and variables in (1) are listed in Tabs. 1 and 2, respectively. By setting $\alpha \ll \beta$, the objective is to first minimize the total number of RSs $I_{\text {total }}$, and secondly minimize the total number of regeneration circuits $(\mathrm{RC})^{1} C_{\text {total }}$. Constraints (1b-1d) are equivalent to $N_{d, i} \leq(1-$ $\left.C_{d, i}\right)\left(N_{d, \operatorname{src}(i ; d)}+L_{d, \operatorname{src}(i ; d), i}\right)$, which calculates the accumulated noise of demand $d$ on link $i \in P_{d}$. Constraint (1f) imposes that the maximum accumulated PLI noise is less than $N_{\max }$. Constraint $(1 \mathrm{~g})$ ensures that the number of RCs at each $\mathrm{RS}$ is lower than the limit $C_{\max }$. Constraints (1h) and (1i) calculate the total numbers of RCs and RSs, respectively. Formulation (1) is a mixed integer linear programming problem with relatively low complexity, which can be solved for a large number of simulations within a short time.

In the second stage, the likelihood of being selected as an RS is calculated for each network node based on the first stage results. We can use either $I_{i}$ (referred to as RS-based) or $\sum_{d \in D} C_{d, i}$ (RC-based) to calculate the likelihood of being an RS for node $i$. The former method uses the knowledge of a node being an RS, whereas the latter one provides information about how many traffic demands are regenerated. Finally, the network nodes are sorted in descending order of likelihood and the top ones are selected as permanent RSs.

\footnotetext{
${ }^{1}$ The $R C$ is an equipment inside the $R S$ dedicated to the regeneration of one light path. One $\mathrm{RC}$ cannot be shared by
}

\begin{tabular}{l|l}
\multicolumn{1}{c}{$\begin{array}{c}\text { Tab. 1: PARAMETERS } \\
\text { Symbol }\end{array}$} & Meaning \\
\hline \hline$D$ & the set of demands \\
\hline$P_{d}$ & the set of nodes on the path of demand $d \in D$ \\
\hline $\operatorname{src}(i ; d) \in P_{d}$ & $\begin{array}{l}\text { the node on the path of demand } d \in D \text { with node } \\
i \in V \text { as the immediate next node }\end{array}$ \\
\hline$L_{d, \operatorname{src}(i ; d), i}$ & $\begin{array}{l}\text { PLI noise generated for demand } d \in D \text { on the link } \\
\text { from src }(i ; d) \text { to } i, \text { src }(i ; d) \text { and } i \text { in } V, \text { it is calculated } \\
\text { by the GN model }\end{array}$ \\
\hline$N_{\max }$ & the maximum tolerable noise before regeneration \\
\hline$C_{\text {max }}$ & the maximum number of regenerator circuits per RS \\
\hline$M$ & $\begin{array}{l}\text { a number larger than the highest possible accumu- } \\
\text { lated noise of any demand } d \in D\end{array}$ \\
\hline$\alpha$, and $\beta$ & $\begin{array}{l}\text { the weight factor for the total number of regeneration } \\
\text { circuits and RSs in the objective of (1), respectively }\end{array}$ \\
\hline \hline & \multicolumn{1}{c}{ Tab. 2: VARIABLES } \\
\hline Symbol & Meaning \\
\hline \hline$N_{d, i} \in \mathbb{R}$ & $\begin{array}{l}\text { accumulated noise for demand } d \in D \text { at the egress } \\
\text { port of node } i \in V\end{array}$ \\
\hline$C_{d, i} \in\{0,1\}$ & $\begin{array}{l}1 \text { if demand } d \in D \text { needs a regenerator circuit on } \\
\text { node } i \in V, 0 \text { otherwise. }\end{array}$ \\
\hline$I_{i} \in\{0,1\}$ & 1 if node $i \in V$ is used as regenerator site \\
\hline$C_{\text {total }}$ & the total number of regenerator circuits in the network \\
\hline$I_{\text {total }}$ & the total number of RSs in the network \\
\hline \hline
\end{tabular}

\section{Numerical Results}

We evaluate the performance of the proposed method using the Coronet CONUS topology 6 with 75 nodes and 99 bidirectional links. The fiber parameters ${ }^{3}$ are used in our simulations. Polarization-multiplexed quadrature phase shift keying is used. The min-distance routing scheme $^{2}$ is used for all the traffic demands. Allto-all traffic demands, whose bandwidth requests follow a normal distribution with a mean of 200 $\mathrm{GHz}$ and a standard deviation of $50 \mathrm{GHz}$, are generated for all the node pairs ${ }^{2}$. We set $C_{\max }=$ $1000, \alpha=1$ and $\beta=0.0001$ such that RC is optimized only after the optimal RSs are found.

We first compare the number of allocated RSs between the proposed method and the RS planning algorithm ${ }^{2}$ with min-distance routing, which is based on the TR constraint and static traffic demands. According to the GN model and the fiber parameters, the TR is calculated to be $2000 \mathrm{~km}$ and 20 RSs are needed by the benchmark. For the proposed method, the number of necessary RSs depends on the specific traffic demands and accumulated PLI noise in each Monte Carlo simulation. As is shown in Fig. 2, around 12 RSs are needed on average and the maximum number of RSs is 16. The much lower number of RSs is attributed to the accurate PLI noise estimation by the GN model and optimization in (1).

In Fig. 3, the normalized likelihood of being an RS for each node is calculated based on the simulation results. The RS- and RC-based meth-

\footnotetext{
multiple light paths.

${ }^{2}$ Node pairs $(s, d)$ and $(d, s)$ for $s, d \in V$ are different, so there are 5550 node pairs in total in our simulations.
} 


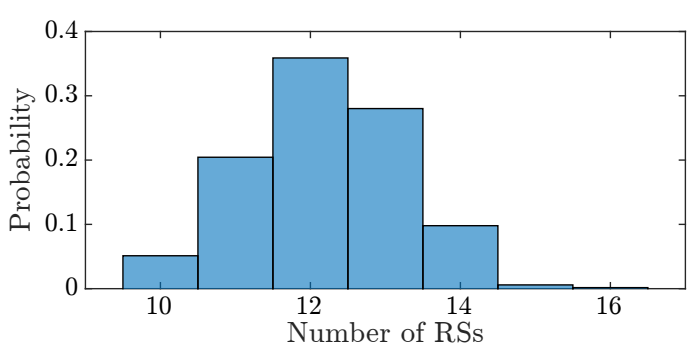

Fig. 2: The histogram of the number of RSs allocated by the proposed method.

ods are compared with the routing only method ${ }^{1}$, which calculates the likelihood of RS for node $i$ by counting the normalized number of demands whose shortest paths go through it. In the RCand RS-based methods, the normalized likelihoods are concentrated to a relatively small subset of nodes, whereas the routing only method assigns a rather uniform distribution for all the nodes.

Tab. 3: RELATIVE GAINS OVER BENCHMARK ${ }^{2}$ IN THE \# OF PROVISIONED DEMANDS AT DIFFERENT BPS

\begin{tabular}{l|l|l} 
BP & RS-based & RC-based \\
\hline \hline 0.005 & $51.4 \% \pm 3.8 \%$ & $67.0 \% \pm 3.2 \%$ \\
\hline 0.01 & $46.2 \% \pm 2.6 \%$ & $62.3 \% \pm 2.1 \%$ \\
\hline 0.02 & $38.7 \% \pm 1.8 \%$ & $56.1 \% \pm 1.4 \%$ \\
\hline 0.04 & $31.9 \% \pm 1.2 \%$ & $47.5 \% \pm 0.9 \%$ \\
\hline
\end{tabular}

The top ranked nodes in Fig. 3 can then be selected as RSs. To compare the blocking performance of the proposed methods with the RS allocation benchmark ${ }^{2}$, the same number (20) of RSs are chosen for both methods. The relative gains in the number of established demands compared with the benchmark and $90 \%$ confidence intervals are shown in Tab. 3. The relative gain is always higher than $30 \%$ for both methods and all BPs.

Tab. 4: RELATIVE GAINS OVER ROUTING-ONLY ${ }^{7}$ IN THE \# OF PROVISIONED DEMANDS AT DIFFERENT RS LIMITS $(B P=0.04)$

\begin{tabular}{l|l|l} 
\#RSs & RS-based & RC-based \\
\hline \hline 10 & $13.2 \%$ & $-4.5 \%$ \\
\hline 15 & $18.7 \%$ & $43.2 \%$ \\
\hline 20 & $24.7 \%$ & $10.1 \%$ \\
\hline 25 & $27.9 \%$ & $14.5 \%$ \\
\hline 30 & $-4.2 \%$ & $10.5 \%$ \\
\hline \hline
\end{tabular}

Finally, the blocking performance of the proposed method with different number of RSs are compared with the routing only method ${ }^{1}$. As is shown in Tab. 4, the proposed methods achieve significant gains for a large range of RS numbers. The $90 \%$ confidence intervals are less than $1 \%$ for all the cases. Note that the gains of the proposed methods are relatively low when the number of RSs are too high or too low. Actually, the proposed method is biased towards the minimum numbers of necessary RSs and RCs. Thus the performance of our algorithm would degenerate when the planned number of RSs disagrees

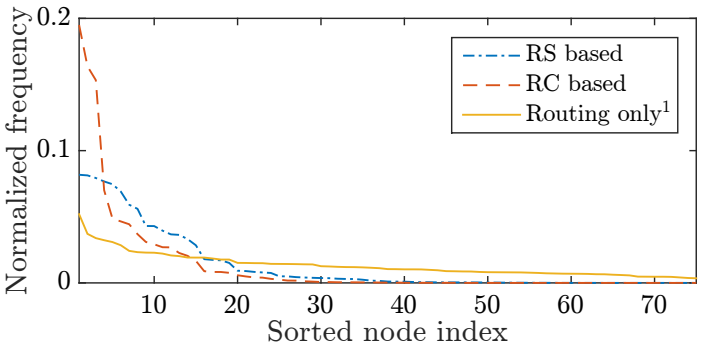

Fig. 3: The normalized likelihood of being selected as RS for network nodes. The nodes are sorted in the descending order of the likelihood.

largely with the optimal one, whose statistics are shown in Fig. 2. However, the blocking performance is still improved from a cost and performance perspective.

\section{Conclusion}

In this paper, an RS assignment algorithm is proposed for dynamic flexible-grid networks considering nonlinear interference and random bandwidth requests. Numerical results demonstrate that significant gains in the number of provisioned demands $(>31 \%)$ is achieved.

\section{Acknowledgements}

This work is supported in part by the NSF Grant CCF-1422871, the Swedish Research Council Grant 2012-5280, and the Ericsson scholarship.

\section{References}

[1] J. Pedro, "Predeployment of regenerators for fast service provisioning in DWDM transport networks," Journal of Optical Communications and Networking, vol. 7, no. 2, pp. A190-A199, 2015.

[2] B. Bathula, A. Chiu, R. Sinha, and S. L. Woodward, "Routing and regenerator planning in a carrier's core ROADM network," in Proc. Optical Fiber Communication Conference (OFC). Los Angeles, CA: Optical Society of America, Mar. 2017, pp. Th4F-4.

[3] N. Dharmaweera, L. Yan, J. Zhao, M. Karlsson, and E. Agrell, "Regenerator site selection in impairmentaware elastic optical networks," in Proc. Optical Fiber Communication Conference (OFC), Anaheim, CA, Mar. 2016, pp. Tu3F-1.

[4] P. Johannisson and E. Agrell, "Modeling of nonlinear signal distortion in fiber-optic networks," IEEE Journal of Lightwave Technology, vol. 32, no. 23, pp. 3942-3950, 2014.

[5] M. Cantono, R. Gaudino, and V. Curri, "Potentialities and criticalities of flexible-rate transponders in DWDM networks: A statistical approach," Journal of Optical Communications and Networking, vol. 8, no. 7, pp. A76A85, 2016.

[6] "CORONET topology." [Online]. Available: http: //monarchna.com/CORONET_CONUS_Topology.xls

[7] F. Pederzolli, D. Siracusa, J. M. Rivas-Moscoso, B. Shariati, E. Salvadori, and I. Tomkos, "Spatial group sharing for SDM optical networks with joint switching," in Proc. IEEE International Conference of Optical Network Design and Modeling (ONDM), Trento, Italy, May 2016, pp. 195-200. 